\title{
A Conformal Averaging Process on the Circle
}

\author{
Richard Evan Schwartz *
}

December 30, 2003

\section{Introduction}

To motivate the main construction in this paper we first describe one of the simplest and best known averaging processes. For some fixed $n \geq 3$ let $X=\left\{x_{1}, \ldots, x_{n}\right\}$ be $n$ distinct and cyclically ordered points on the circle $\boldsymbol{R} / \boldsymbol{Z}$. Define $X^{\prime}=\left\{x_{1}^{\prime}, \ldots, x_{n}^{\prime}\right\}$ where $x_{j}^{\prime}$ is the midpoint of the segment $I_{j}$ whose endpoints are $x_{j}$ and $x_{j+1}$, with indices (as always) taken mod $n$. Of the two possibilities, $I_{j}$ is chosen so that it contains no other $x_{k}$. In general let $X^{(k+1)}=\left(X^{(k)}\right)^{\prime}$. To understand the asymptotic behavior of $\left\{X^{(k)}\right\}$ one defines the energy

$$
\varphi(X)=\sum_{j=1}^{n}\left|I_{j}\right|^{2} .
$$

Here $\left|I_{j}\right|$ is the length of the interval $I_{j}$. The minimum value, $1 / n$, occurs when the points are evenly spaced-that is, when $\left|I_{1}\right|=\ldots=\left|I_{n}\right|$. Using the fact that $\left|I_{j}^{\prime}\right|=\frac{1}{2}\left(\left|I_{j}\right|+\left|I_{j+1}\right|\right)$ we get, after some algebra:

$$
\varphi(X)-\varphi\left(X^{\prime}\right)=\frac{1}{4} \sum_{i=1}^{n}\left(\left|I_{j}\right|-\left|I_{j+1}\right|\right)^{2} \geq 0
$$

Thus we see that $\varphi\left(X^{\prime}\right) \leq \varphi(X)$ with equality iff the points are evenly spaced. Moreover, the difference $\varphi(X)-\varphi\left(X^{\prime}\right)$ is large unless the points of $X$ are nearly evenly spaced. From these two observations we see that the points of $\left\{X^{(k)}\right\}$ rapidly become evenly spaced as $k \rightarrow \infty$.

\footnotetext{
* Supported by N.S.F. Grant DMS-0305047 and also by a Guggenheim Fellowship
} 
The averaging process we just considered uses the metric structure of $\boldsymbol{R} / \boldsymbol{Z}$ for its definition. In this paper we will consider the simplest kind of averaging process that is based on the conformal structure of the unit circle $S^{1}$, considered as the ideal boundary of the hyperbolic plane $\boldsymbol{H}^{2}$. See [B] for basic information about $\boldsymbol{H}^{2}$. The natural automorphisms of $S^{1}$, the conformal transformations, are then interpreted as extensions to the ideal boundary of isometries of $\boldsymbol{H}^{2}$.

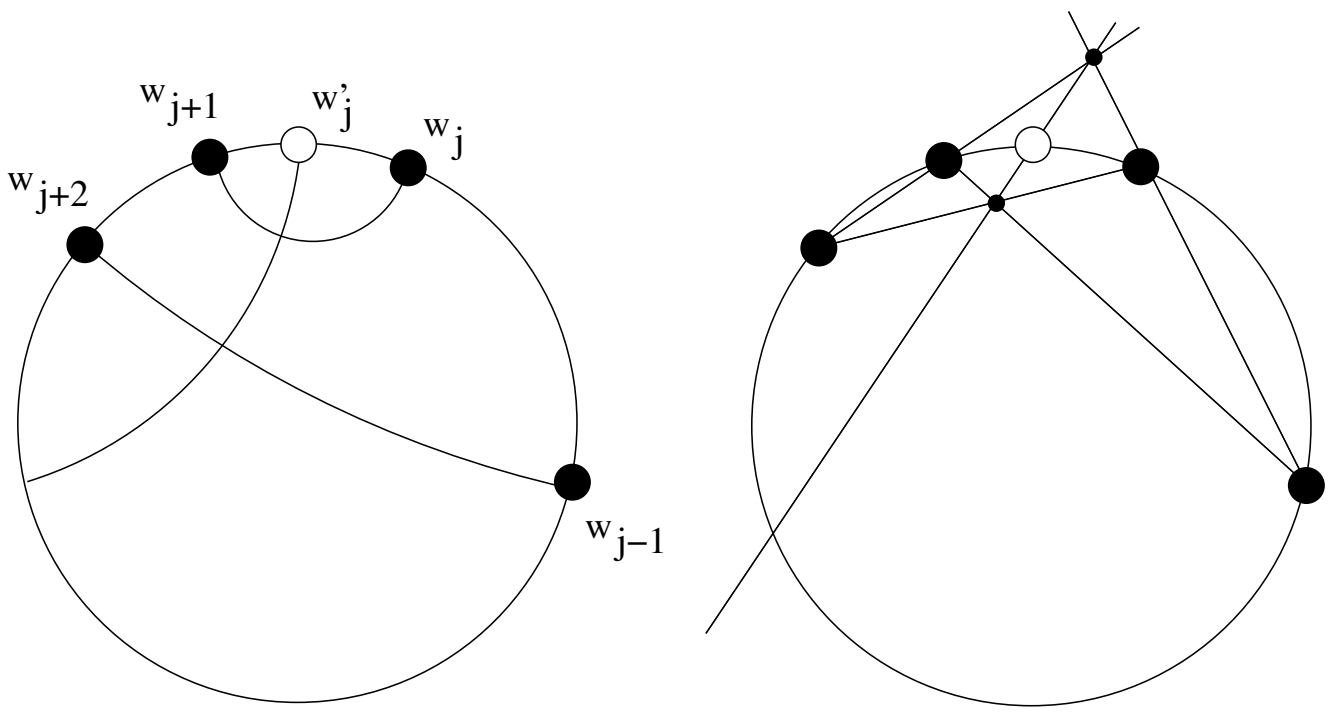

Figure 1

Let $n \geq 5$. Let $W=\left\{w_{1}, \ldots, w_{n}\right\} \subset S^{1}$ be a list of $n$ cyclically ordered points-i.e. an $n$-gon. Let $I_{j}$ be as above. We define $w_{j}^{\prime} \in I_{j}$ by either of the constructions shown in Figure 1. The first construction, which is adapted to the Poincaré model of $\boldsymbol{H}^{2}$, involves a configuration of circles, in which every two circles are either disjoint or intersect at right angles. The second construction, which is adapted to the Klein model of $\boldsymbol{H}^{2}$, involves straight lines. Let $W^{\prime}=\left\{w_{1}^{\prime}, \ldots, w_{n}^{\prime}\right\}$. We call $W^{\prime}$ the conformal average of $W$. The process is conformally natural: If $h$ is a conformal transformation then $(h(W))^{\prime}=h\left(W^{\prime}\right)$. We define $W^{(k+1)}=\left(W^{(k)}\right)^{\prime}$. We are interested in the asymptotic behavior of the sequence $\left\{W^{(k)}\right\}$-which is to say, of the conformal averaging process.

Say that $W$ is conformally regular if there is a conformal transformation $h$ such that $h(W)$ is the vertex set of a regular $n$-gon. Here is our main result: 
Theorem 1.1 For any $n$-gon $W$, there is a conformally regular $n$-gon $W^{(\infty)}$ such that $\left\{W^{(2 k)}\right\}$ converges exponentially fast to $W^{(\infty)}$.

By exponentially fast we mean that there are positive constants $C_{1}, C_{2}$, depending on $W$, such that every vertex of $W^{(2 k)}$ is within $C_{1} \exp \left(-C_{2} k\right)$ of the corresponding vertex of $W^{(\infty)}$. The sequence $\left\{W^{(2 k+1)}\right\}$ likewise converges exponentially fast to $\left(W^{(\infty)}\right)^{\prime}$, and $\left(W^{\infty}\right)^{\prime \prime}=W^{\infty}$.

To prove Theorem 1.1 we introduce a conformally natural energy function which increases under the conformal averaging process. Given 4 distinct unit complex numbers $a, b, c, d \in S^{1}$ we have their cross ratio

$$
\chi(a, b, c, d)=\frac{(a-b)(c-d)}{(a-c)(b-d)}
$$

If $a, b, c, d$ come in cyclic order on $S^{1}$ then $\chi(a, b, c, d) \in(0,1)$. See $[\mathbf{B}]$. We define

$$
\phi(W)=\prod_{j=1}^{n} z_{j} ; \quad z_{j}=\chi\left(w_{j}, w_{j+1}, w_{j+2}, w_{j+3}\right) .
$$

We will see that

$$
\phi\left(W^{\prime}\right) \geq \phi(W)
$$

with equality iff $W$ is conformally regular. We will also see that $\phi\left(W^{\prime}\right)-\phi(W)$ is large if $W$ is far from being conformally regular. These properties are the main ingredients in our proof.

The conformal averaging process is a discrete analogue of a P.D.E. we studied in $[\mathbf{S}]$. Our main result ${ }^{1}$ there is an analogue of Theorem 1.1 for circle diffeomorphisms. The main idea there is to establish the monoticity of the integral of the Schwarzian derivative, which is a smooth analogue of the functional $\log \phi$. The work in $[\mathbf{S}]$ was motivated by the conformal averaging process. We would have liked to prove Theorem 1.1 at the time we wrote $[\mathbf{S}]$, but we didn't know to do it.

Equation 5 has a nice consequence.

Corollary 1.2 Suppose $W_{1}$ and $W_{2}$ are two $n$-gons in $S^{1}$, with $W_{2}$ being conformally regular. Then $\phi\left(W_{1}\right) \leq \phi\left(W_{2}\right)$, with equality iff $W_{1}$ is also conformally regular.

\footnotetext{
${ }^{1}$ It seems worthwhile to point out an embarrassing typo we noticed, long after the publication, in the statement of the main theorem in $[\mathbf{S}]$. The derivative $\partial / \partial t^{n}$ should (obviously) be $\partial / \partial x^{n}$.
} 
Equation 5 is an immediate consequence of the following two equations:

$$
\begin{gathered}
\frac{\phi\left(W^{\prime}\right)}{\phi(W)}=\left[\frac{\prod_{i=1}^{n}\left(1+a_{i}+a_{i+1}\right)}{\prod_{i=1}^{n}\left(\left(1+a_{i-1}\right)\left(1+a_{i+1}\right)-a_{i}^{2}\right)}\right]^{2} ; \quad a_{j}=\sqrt{z_{j} .} \\
\prod_{i=1}^{n}\left(1+a_{i}+a_{i+1}\right) \geq \prod_{i=1}^{n}\left(\left(1+a_{i-1}\right)\left(1+a_{i+1}\right)-a_{i}^{2}\right) \quad \forall\left(a_{1}, \ldots, a_{n}\right) \in[0,1]^{n} .
\end{gathered}
$$

We have equality iff $a_{1}=\ldots=a_{n}$. As usual, indices are taken $\bmod n$.

We found Equation 7 too hard to tackle directly, so we split it up as:

$$
\begin{gathered}
\prod_{i=1}^{n}\left(1+a_{i}+a_{i+1}\right) \geq \prod_{i=1}^{n}\left(1+2 a_{i}\right) \quad \forall\left(a_{1}, \ldots, a_{n}\right) \in[0,1]^{n} ; \\
\prod_{i=1}^{n}\left(1+2 a_{i}\right) \geq \prod_{i=1}^{n}\left(\left(1+a_{i-1}\right)\left(1+a_{i+1}\right)-a_{i}^{2}\right) \quad \forall\left(a_{1}, \ldots, a_{n}\right) \in[0,1]^{n} .
\end{gathered}
$$

In both cases we have equality iff $a_{1}=\ldots=a_{n}$. Equation 8 has an easy proof. We tried hard to find an easy proof of Equation 9, but couldn't do it. We reduce Equation 9 to the statement that a certain polynomial $P \in Z[\sqrt{5}](X, Y, Z)$ is non-negative on the unit cube; then we prove it. $P$ has over 500 terms, both positive and negative, but the positive terms dominate the negative terms in a certain sense. If Equation 9 has a simple proof we would love to see it.

From the point of view of conformal geometry there is more than one circle. Say that a conformal circle is a smooth circle-i.e. a smooth connected closed 1-manifold-equipped with a system of coordinate charts into $S^{1}$ such that the overlap functions are restrictions of conformal maps. Such an object is also called an $\boldsymbol{R P}^{1}$-structure on a circle. Two conformal circles are equivalent if there is a diffeomorphism between them, which is locally conformal when measured in the special coordinate systems. The set of inequivalent conformal circles is bijective with the set of conjugacy classes of elements in $\widetilde{S L_{2}}(\boldsymbol{R})$, the universal cover of $S L_{2}(\boldsymbol{R})$. See $[\mathbf{K}]$ and also $\S 6$ for more details.

If $S$ is a conformal circle and $w \in S$ is a point then there is a locally conformal map $\operatorname{dev}_{w}: S-\{w\} \rightarrow S^{1}$, unique up to composition with a conformal automorphism of $S^{1}$. We call $\mathbf{d e v}_{w}$ a local developing map. We call $S$ small if $\mathbf{d e v}_{w}$ is injective and omits more than one point, for every $w \in S$. We call $S$ medium if $S$ is not small but $\mathbf{d e v}_{w}$ is injective for all 
$w \in S$. Otherwise we call $S$ large. When $S$ is small or medium we can use the local developing maps to define the conformal averaging process for polygons in $S$.

When $S$ is small, $S$ has a canonical Riemannian metric, invariant under its whole conformal automorphism group. In this case, it makes sense to speak of regular $n$-gons on $S$.

Theorem 1.3 Suppose $S$ is small. For any $n$-gon $W \subset S$, there is a regular $n$-gon $W^{(\infty)} \subset S$ such that $\left\{W^{(2 k)}\right\}$ converges exponentially fast to $W^{(\infty)}$.

Aside from $S^{1}$ there is one other medium conformal circle, which we call $S_{-}^{1}$. This circle corresponds to a certain element of $\widetilde{S L_{2}}(\boldsymbol{R})$ which covers a parabolic. $S_{-}^{1}$ is a funny point in the moduli space of all conformal circles; $S^{1}$ and $S_{-}^{1}$ cannot be placed inside disjoint open sets. We think of $S_{-}^{1}$ as being "infinitesimally smaller than" $S^{1}$. Let $\mathcal{W}\left(S_{-}^{1}, n\right)$ denote the space of $n$-gons in $S_{-}^{1}$.

Theorem 1.4 For any $n$-gon $W \subset S_{-}^{1}$, the sequence $\left\{W^{(k)}\right\}$ exits every compact subset of $\mathcal{W}\left(S_{-}^{1}, n\right)$. At the same time, the list of cross ratios of $W^{(k)}$ converges to the list of cross ratios for the regular $n$-gon.

We shall not discuss all the large conformal circles, but will concentrate on the simplest examples. Let $\theta>1$. The large conformal circle $S_{\theta}^{1}$ has the following description. Let $\widetilde{S}^{1}$ denote the universal cover of $S^{1}$ and let $T_{\theta}$ be the isomorphism of $\widetilde{S}^{1}$ which translates by $2 \pi \theta$. Then $S_{\theta}^{1}=\widetilde{S}^{1} / T_{\theta}$. When $\theta$ is an integer, this definition coincides with the usual definition of a covering space. A regular $n$-gon in $S_{\theta}^{1}$ is an $n$-gon whose list of cross ratios is constant. These are unique up to conformal automorphism of $S_{\theta}^{1}$.

It turns out that the conformal averaging process is not defined for every $n$-gon in a large conformal circle, as we explain in $\S 6.3$. Let $\mathcal{W}\left(S_{\theta}^{1}, n\right)$ denote the space of $n$-gons in $S_{\theta}^{1}$ for which all the cross ratios are defined. This condition means that 4 consecutive points of $W$ must lie in an arc of $S_{\theta}^{1}$ of length less than $2 \pi$, as measured in a canonical Riemannian metric on $S_{\theta}^{1}$. See $\S 6.3$ for more details. The conformal averaging process is only defined for polygons in $\mathcal{W}\left(S_{\theta}^{1}, n\right)$.

Let $n>4 \theta$. As we will see in $\S 6.4$, this condition guarantees that the regular $n$-gons in $S_{\theta}^{1}$ belong to $\mathcal{W}\left(S_{\theta}^{1}, n\right)$. We call a polygon $W \subset \mathcal{W}\left(S_{\theta}^{1}, n\right)$ excessive if $\phi(W)>\phi\left(W_{0}\right)$, where $W_{0}$ is a regular $n$-gon in $S_{\theta}^{1}$. 
Theorem 1.5 Let $\theta>1$ and $n>4 \theta$. Suppose that $W \in \mathcal{W}\left(S_{\theta}^{1}, n\right)$ is excessive. Then there is some $k$ such that $W^{(k)}$ is not defined.

For excessive polygons, the conformal averaging process relentlessly disorders the points until the process can no longer be defined. Theorem 1.5 is similar to the "finite time blow-up" result of $[\mathbf{S}]$. Incidentally, to show that Theorem 1.5 is not vacuous, we prove:

Theorem 1.6 Let $\theta>1$ and $n>4 \theta$. Then $\mathcal{W}\left(S_{\theta}^{1}, n\right)$ contains excessive $n$-gons which are arbitrarily close to being regular.

We think that the contrasting statements in Theorems 1.1, 1.3, 1.4 and 1.5 have more to do with the nature of conformal circles than they have to do with the specific averaging process. For instance, the main structural difference between $S^{1}$ and $S_{\theta}^{1}$, which impacts the conformal averaging process, is this: The regular polygons in $S^{1}$ are maxima for $\phi$ but the regular polygons in $S_{\theta}^{1}$ are saddle points for $\phi$. Any non-trivial conformally natural process which satisfies Equation 5 should behave like the process we consider. To illustrate this principle, we will consider a modified process in $\S 7$ and reprove all our results for it. The modified process is less symmetric than the conformal averaging process, but its analysis avoids the pain of Equation 9 and thereby gives a swifter proof of Corollary 1.2.

We discovered the mathematics in this paper by experimenting on the computer. Accordingly, our proofs sometimes require some symbolic manipulation, which we perform in Mathematica $[\mathbf{W}]$. Specifically,

1. We take the cross ratio of the 4 quantities in Equation 17 and simplify the result to get Equation 19. Similarly, we take the cross ratio of the 4 quantities in Equation 69 and simplify the result to get Equation 70.

2. We compute some first and second partial derivatives of the function $\phi$ (Equation 4), the function $E$ (Equation 28), the function $F$ (Equation 33 ), and the function $G$ (Equation 73).

3. We compute the quantities $\gamma$ and $\delta$ in Equation 47.

4. We compute the polynomial $P(X, Y, Z)$ mentioned above, and also the auxilliary polynomials $P_{0}, \widehat{P}_{0}, P_{1}, P_{2}, P_{3}, \widehat{P}_{3}, \Pi_{1}, \Pi_{2}$ defined in $\S 4.3$. 
Someone who has a rudimentary knowledge of Mathematica can easily reproduce Computations 1 and 2, and the right sort of reader can do them by hand. For the convenience of the reader, we include in $\S 4.4$ our computer code for Computations 3 and 4 . This code can be copied (back) into a file verbatim and run in Mathematica.

Alternatively, the reader can verify our calculations using the files in the package www.math.umd.edu/ res/Papers/conformal.tar. Once downloaded, the package can be unpacked on a unix machine with the command: tar - xvf conformal.tar. This command produces the directory Conformal, which has the relevant files.

This paper is organized as follows.

- In $\S 2$ we prove Equation 6.

- In $\S 3$ we prove Equation 8.

- In $\S 4$ we prove Equation 9.

- In $\S 5$ we prove Theorem 1.1 and Corollary 1.2.

- In $\S 6$ we prove Theorems $1.3,1.4,1.5$, and 1.6.

- In $\S 7$ we reprove all our results for a modified process.

I thank Jeremy Kahn, whose insightful comments streamlined the method in $§ 4.3$. I also thank the John Simon Guggenheim Memorial Foundation, the National Science Foundation, the University of Maryland, and the Institute for Advanced Study, for their generous support. 


\section{Proof of Equation 6}

\subsection{The Derivation}

Stereographic projection gives a conformal isomorphism between $S^{1}$, the circle of unit complex numbers, and $\boldsymbol{R} \cup \infty$, the real projective line. See $[\mathbf{B}]$. In this chapter we will use this isomorphism, so that our computations take place in $\boldsymbol{R} \cup \infty$. This makes life a bit easier for us. The interested reader can check our computations in this chapter using our file mapping.

For our derivation we consider sequences $w_{-1}, w_{0}, w_{1}, \ldots, w_{n}$ where

$$
w_{-1}=\infty ; \quad w_{0}=0 ; \quad w_{1}=1 ; \quad w_{1}<w_{2}<\ldots<w_{n}<\infty .
$$

Given $\chi$ as in Equation 3 we define

$$
z_{j}=\chi\left(w_{j-2}, w_{j-1}, w_{j}, w_{j+1}\right) ; \quad j=1,2,3, \ldots
$$

First we explain how to reconstruct the $w$ variables in terms of the $z$ variables.

Say that a finite subset $I \subset \boldsymbol{Z}$ is admissible if it does not contain any consecutive integers. Let $A$ denote the set of nonempty admissible subsets. Let $|I|$ denote the cardinality of $I \in A$. Define the formal series

$$
S=S\left(\ldots, z_{1}, z_{2}, z_{3}, \ldots\right)=1+\sum_{I \in A} m_{I} ; \quad m_{I}=(-1)^{|I|} \prod_{i \in I} z_{i}
$$

Here is the reconstruction formula:

$$
w_{j}=\frac{S\left(\ldots, 0,0,0,0, z_{2}, \ldots, z_{j-1}, 0,0,0 \ldots\right)}{S\left(\ldots, 0,0,0, z_{1}, z_{2}, \ldots, z_{j-1}, 0,0,0 \ldots\right)} ; \quad j=2,3,4 \ldots
$$

(When $j=2$ the notation needs to be suitably interpreted, as in Equation 14.) The first few terms are:

$$
\begin{gathered}
w_{2}=\frac{1}{1-z_{1}} ; \\
w_{3}=\frac{1-z_{2}}{1-z_{1}-z_{2}} ; \\
w_{4}=\frac{1-z_{2}-z_{3}}{1-z_{1}-z_{2}-z_{3}+z_{1} z_{3}} ; \\
w_{5}=\frac{1-z_{2}-z_{3}-z_{4}+z_{2} z_{4}}{1-z_{1}-z_{2}-z_{3}-z_{4}+z_{1} z_{3}+z_{1} z_{4}+z_{2} z_{4}} .
\end{gathered}
$$


Technically, we only need the formulas in Equation 14, and these can be verified by a symbolic manipulator. For the interested reader, we prove Equation 13 in $\S 2.2$.

Uur basic construction in $\S 1$ produces a point $w_{j}^{\prime}$ between the points $w_{j}$ and $w_{j+1}$. By symmetry, the point $w_{j}^{\prime}$ is determined, up to taking the branch of the square root function, by the equality:

$$
\chi\left(w_{j-1}, w_{j}, w_{j}^{\prime}, w_{j+1}\right)=\chi\left(w_{j}, w_{j}^{\prime}, w_{j+1}, w_{j+2}\right) .
$$

After some fooling around, we discovered that

$$
w_{j}^{\prime}=\frac{S\left(\ldots, 0,0,0,0, z_{2}, \ldots, z_{j},-\sqrt{z_{j+1}}, 0,0,0 \ldots\right)}{S\left(\ldots, 0,0,0, z_{1}, z_{2}, \ldots, z_{j},-\sqrt{z_{j+1}}, 0,0,0 \ldots\right)} .
$$

(When $j=0,1$ the formula needs to be suitably interpreted, as in Equation 17.) The first few terms are:

$$
\begin{gathered}
w_{0}^{\prime}=\frac{1}{1+\sqrt{z_{1}}} ; \\
w_{1}^{\prime}=\frac{1+\sqrt{z_{2}}}{1+\sqrt{z_{2}}-z_{1}} ; \\
w_{2}^{\prime}=\frac{1-z_{2}+\sqrt{z_{3}}}{1-z_{1}-z_{2}+\sqrt{z_{3}}-z_{1} \sqrt{z_{3}}} ; \\
w_{3}^{\prime}=\frac{1-z_{2}-z_{3}+\sqrt{z_{4}}-z_{2} \sqrt{z_{4}}}{1-z_{1}-z_{2}-z_{3}+\sqrt{z_{4}}+z_{1} z_{3}-z_{1} \sqrt{z_{4}}-z_{2} \sqrt{z_{4}}} .
\end{gathered}
$$

In all cases, the positive branch of the square root is taken. Technically, we just need Equation 17, and these 4 formulas can be verified by a symbolic manipulator. For the interested reader, we will prove Equation 16 in $§ 2.3$

Let

$$
z_{j}^{\prime}=\chi\left(w_{j-1}^{\prime}, w_{j}^{\prime}, w_{j+1}^{\prime}, w_{j+2}^{\prime}\right) ; \quad j=1,2,3, \ldots
$$

Plugging Equation 17 into Mathematica we compute (in the file mapping) that

$$
z_{1}^{\prime}=\frac{a_{2} a_{3}\left(1+a_{1}+a_{2}\right)\left(1+a_{3}+a_{4}\right)}{\left[\left(1+a_{1}\right)\left(1+a_{3}\right)-a_{2}^{2}\right]\left[\left(1+a_{2}\right)\left(1+a_{4}\right)-a_{3}^{2}\right]} ; \quad a_{j}=\sqrt{z_{j}} .
$$

By symmetry, the formula for $z_{i}^{\prime}$ is obtained from Equation 19 by shifting all the indices by $i-1$. Taking the product of the $z_{i}^{\prime}$, with indices taken $\bmod n$, gives Equation 6. 


\subsection{Proof of Equation 13}

For ease of exposition we will take $j \geq 4$ so as to avoid having to use special notation for the first few cases. Let $N_{j}$ and $D_{j}$ denote the numerator and denominator respectively in Equation 13. First of all, one can verify the identities

$$
N_{j}=N_{j-1}-z_{j-1} N_{j-2} ; \quad D_{j}=D_{j-1}-z_{j-1} D_{j-2}
$$

directly from the definitions. The basic idea is that the above recurrence relations never introduce monomials which involve consecutive variables, but do introduce all other monomials, and with the correct signs.

From Equation 20 we have

$$
\begin{gathered}
N_{j-2} D_{j}-D_{j-2} N_{j}= \\
N_{j-2}\left(D_{j-1}-z_{j-1} D_{j-2}\right)-D_{j-2}\left(N_{j-1}-z_{j-1} N_{j-2}\right)= \\
N_{j-2} D_{j-1}-D_{j-2} N_{j-1}
\end{gathered}
$$

and

$$
\begin{gathered}
N_{j} D_{j+1}-D_{j} N_{j+1}= \\
N_{j}\left(D_{j}-z_{j} D_{j-1}\right)-D_{j}\left(N_{j}-z_{j} N_{j-1}\right)= \\
z_{j}\left(N_{j-1} D_{j}-D_{j-1} N_{j}\right) .
\end{gathered}
$$

We have

$$
\begin{gathered}
\chi\left(w_{j-2}, w_{j-1}, w_{j}, w_{j+1}\right)= \\
\chi\left(\frac{N_{j-2}}{D_{j-2}}, \frac{N_{j-1}}{D_{j-1}}, \frac{N_{j}}{D_{j}}, \frac{N_{j+1}}{D_{j+1}}\right)=^{*} \\
\frac{N_{j-2} D_{j-1}-D_{j-2} N_{j-1}}{N_{j-2} D_{j}-D_{j-2} N_{j}} \times \frac{N_{j} D_{j+1}-D_{j} N_{j+1}}{N_{j-1} D_{j+1}-D_{j-1} N_{j+1}}= \\
1 \times \frac{z_{j}\left(N_{j-1} D_{j}-D_{j-1} N_{j}\right)}{N_{j-1} D_{j+1}-D_{j-1} N_{j+1}}= \\
z_{j} .
\end{gathered}
$$

The starred equality just boils down to clearing denominators. The subsequent equalities are consequences of Equations 22 and 21. 


\subsection{Proof of Equation 16}

For ease of exposition we will take $j \geq 3$ so as to avoid having to use special notation for the first few cases. Let $\widetilde{N}_{j}$ and $\widetilde{D}_{j}$ denote the numerator and denominator of the fraction in Equation 16.

From Equation 20 we get

$$
\widetilde{N}_{j}=N_{j+1}+\sqrt{z_{j+1}} N_{j} ; \quad \widetilde{D}_{j}=D_{j+1}+\sqrt{z_{j+1}} D_{j} .
$$

This equation gives us

$$
\begin{gathered}
\widetilde{N}_{j} D_{j+1}-\widetilde{D}_{j} N_{j+1}= \\
\left(N_{j+1}+\sqrt{z_{j+1}} N_{j}\right) D_{j+1}-\left(D_{j+1}+\sqrt{z_{j+1}} D_{j}\right) N_{j+1}= \\
\sqrt{z_{j+1}}\left(N_{j} D_{j+1}-D_{j} N_{j+1}\right)
\end{gathered}
$$

and

$$
\begin{gathered}
N_{j-1} \widetilde{D}_{j}-D_{j-1} \widetilde{N}_{j}= \\
N_{j-1}\left(D_{j+1}+\sqrt{z_{j+1}} D_{j}\right)-D_{j-1}\left(N_{j+1}+\sqrt{z_{j+1}} N_{j}\right)= \\
\left(N_{j-1} D_{j+1}-D_{j-1} N_{j+1}\right)+\sqrt{z_{j+1}}\left(N_{j-1} D_{j}-D_{j-1} N_{j}\right)={ }^{*} \\
\left(1+\sqrt{z_{j+1}}\right)\left(N_{j-1} D_{j}-D_{j-1} N_{j}\right) .
\end{gathered}
$$

The starred equality comes from Equation 21. Let $A=\chi\left(w_{j-1}, w_{j}, w_{j}^{\prime}, w_{j+1}\right)$ and $B=\chi\left(w_{j}, w_{j}^{\prime}, w_{j+1}, w_{j+2}\right)$. We want to prove that $A=B$. Using the above equations, we compute

$$
\begin{gathered}
A=\chi\left(\frac{N_{j-1}}{D_{j-1}}, \frac{N_{j}}{D_{j}}, \frac{\widetilde{N}_{j}}{\widetilde{D}_{j}}, \frac{N_{j+1}}{D_{j+1}}\right)= \\
\frac{N_{j-1} D_{j}-D_{j-1} N_{j}}{N_{j-1} \widetilde{D}_{j}-D_{j-1} \widetilde{N}_{j}} \times \frac{\widetilde{N_{j}} D_{j+1}-\widetilde{D}_{j} N_{j+1}}{N_{j} D_{j+1}-D_{j} N_{j+1}}= \\
\frac{1}{1+\sqrt{z_{j+1}}} \times \sqrt{z_{j+1}}=\frac{\sqrt{z_{j+1}}}{1+\sqrt{z_{j+1}}} .
\end{gathered}
$$

A similar computation gives the same result for $B$, but we will finish the proof in a different way. Let $f(x)=x /(1-x)$. Note that $f(A)=\sqrt{z_{j+1}}$. For any 5 points $a, b, c, d$, e there is the general, and easily checked, identity

$$
f(\chi(a, b, c, d)) \times f(\chi(b, c, d, e))=\chi(a, b, d, e) .
$$

This equality gives us, a priori, that $f(A) f(B)=z_{j+1}$. Since $f(A)=\sqrt{z_{j+1}}$ we must also have $f(B)=\sqrt{z_{j+1}}$. But then $f(A)=f(B)$ and hence $A=B$, as claimed. 


\section{Proof of Equation 8}

\subsection{The Proof}

Define

$$
E\left(a_{1}, \ldots, a_{n}\right)=\log \frac{\prod_{i=1}^{n}\left(1+a_{i}+a_{i+1}\right)}{\prod_{i=1}^{n}\left(1+2 a_{i}\right)}
$$

Equation 8 is true iff the restriction of $E$ to $(0,1)^{n}$ is minimized precisely at the diagonal.

Consider a sequence $A=(\ldots a, b, c \ldots)$. Let $E_{b}=\partial E / \partial b$. We compute

$$
E_{b}(A)=\frac{\left(2 b^{2}+2 b\right)-(2 a c+a+c)}{(1+2 b)(1+a+b)(1+b+c)} .
$$

See the file gradient in our package. If $A$ does not lie on the diagonal we can arrange that $\max (a, c) \leq b$ and $\min (a, c)<b$. This forces $E_{b}(A)>0$. But then we can decrease $E(A)$ by slightly decreasing $b$. This shows that $E$ attains its global minimum precisely along the diagonal.

\subsection{An Extra Estimate}

Here we prove an estimate for Equation 8 which, later on, will quantify the statement that $\phi\left(W^{\prime}\right)-\phi(W)$ is large when $W$ is far from conformally regular. Incidentally, a similar estimate is also true-and fairly easy to prove-for Equation 9 as well. However, for our purposes it suffices to prove the result for Equation 8.

Let $\Delta$ be the diagonal in $(0,1)^{n}$. Let $A \in \Delta$. Let $N_{\epsilon}(A)$ be the $\epsilon$ neighborhood of $A$. If $\epsilon$ is small then $N_{\epsilon}(A) \subset(0,1)^{n}$ and $E$ is everywhere defined on $N_{\epsilon}(A)$. Given any $q \in(0,1)^{n}$ let $\delta(q)$ denote the Euclidean distance from $q$ to $\Delta$.

Lemma 3.1 For any $A \in \Delta$ there are positive constants $C=C(A)$ and $\epsilon=\epsilon(A)$ with the following property: If $q \in N_{\epsilon}(A)$ then $E(q) \geq C(\delta(q))^{2}$.

Proof: It follows from symmetry that $\nabla E(A)=0$ when $A=(a, \ldots, a) \in \Delta$. Let $H_{E}(A)$ denote the Hessian of second partial derivatives of $E$, evaluated at $A$. Let $\Delta^{\perp}$ denote the subspace of $\boldsymbol{R}^{n}$ which is perpendicular to $\Delta$. We will show, for all $A \in \Delta$, that $H_{E}(A)$ is positive definite when restricted to $\Delta^{\perp}$. Lemma 3.1 follows from these two facts by integration. For ease of 
notation we will take $n=7$ in our computation. The reader will be able to see the obvious pattern from this case.

We compute that

$$
E_{a b}=\frac{-1}{(1+2 a)^{2}} ; \quad E_{b b}=\frac{2}{(1+2 a)^{2}} ; \quad E_{c b}=\frac{-1}{(1+2 a)^{2}} .
$$

Here we have set $A=(\ldots a, b, c \ldots)=(\ldots a, a, a \ldots)$. This computation works for every coordinate, so that

$$
H_{E}(A)=(1+2 a)^{-2}\left[\begin{array}{ccccccc}
2 & -1 & 0 & 0 & 0 & 0 & -1 \\
-1 & 2 & -1 & 0 & 0 & 0 & 0 \\
0 & -1 & 2 & -1 & 0 & 0 & 0 \\
0 & 0 & -1 & 2 & -1 & 0 & 0 \\
0 & 0 & 0 & -1 & 2 & -1 & 0 \\
0 & 0 & 0 & 0 & -1 & 2 & -1 \\
-1 & 0 & 0 & 0 & 0 & -1 & 2
\end{array}\right]
$$

The eigenvalues of $H_{E}(A)$ are

$$
(1+2 a)^{-2}(2-2 \cos (2 \pi k / 7)) ; \quad k=0, \ldots, 6 .
$$

The 0 -eigenvalue corresponds to the eigenvector $(1,1,1,1,1,1,1)$. The remaining eigenvectors, which correspond to positive eigenvalues, span $\Delta^{\perp}$. This completes the proof of Lemma 3.1. 


\section{Proof of Equation 9}

\subsection{Equation 9 Reduced to Lemma 4.2}

We assume $n \geq 5$ in our proof. (The case $n=12$ implies the cases $n=2,3,4$.) Let

$$
F\left(a_{1}, \ldots, a_{n}\right)=\log \prod_{i=1}^{n}\left(\left(1+a_{i-1}\right)\left(1+a_{i+1}\right)-a_{i}^{2}\right)-\log \prod_{i=1}^{n}\left(1+2 a_{i}\right)
$$

To prove Equation 9 it suffices to prove that $F$, restricted to $(0,1)^{n}$, attains its maximum-namely 0 -precisely on the diagonal.

We consider $F(a, b, c, d, e, \ldots)$. Using the file gradient we take some partial derivatives:

$$
\begin{gathered}
F_{c}=\frac{-2}{1+2 c}-\frac{2 c}{(1+b)(1+d)-c^{2}}+\frac{1+a}{(1+a)(1+c)-b^{2}}+\frac{1+e}{(1+e)(1+c)-d^{2}} . \\
F_{a c}=-\frac{b^{2}}{\left((1+a)(1+c)-b^{2}\right)^{2}} \leq 0 \\
F_{b c}=\frac{2 b(1+a)}{\left((1+a)(1+c)-b^{2}\right)^{2}}+\frac{2 c(1+d)}{\left((1+b)(1+d)-c^{2}\right)^{2}} \geq 0 . \\
F_{d c}=\frac{2 c(1+b)}{\left((1+b)(1+d)-c^{2}\right)^{2}}+\frac{2 d(1+e)}{\left((1+c)(1+e)-d^{2}\right)^{2}} \geq 0 . \\
F_{e c}=-\frac{d^{2}}{\left((1+e)(1+c)-d^{2}\right)^{2}} \leq 0 .
\end{gathered}
$$

The denominators do not vanish when $(a, b, c, d, e) \in(0,1)^{5}$. More generally, these equations make sense for $(a, b, c, d, e) \in \boldsymbol{R}^{5}$ as long as the denominators do not vanish. Compare Lemma 4.5 and Corollary 4.10 below.

Note that $F_{d c}>0$ if $d>0$. Suppose that $d>2 c$. From Equation 35 and (for the last equality) some algebra we have

$$
\begin{gathered}
F_{c}(a, b, c, d, e)>F_{c}(a, b, c, 2 c, e) \geq F_{c}(1, b, c, 2 c, 1) \geq F_{c}(1,0, c, 2 c, 1)= \\
\frac{4 c^{2}}{(1+c)(1-c)(1+2 c)\left(1+2 c-c^{2}\right)} \geq 0 .
\end{gathered}
$$

In short, if $d>2 c$ we can increase $F$ by increasing $c$ and keeping $c<1$. By symmetry, if $c>2 d$ be can increase $F$ by increasing $d$ and keeping $d<1$. 
Let $[0,1]_{2}^{n}$ denote those $\left(a_{1}, \ldots, a_{n}\right) \in[0,1]^{n}$ such that $a_{i} / a_{i+1} \in[1 / 2,2]$ for all $i$. Note that Equations 33, 34, and 35 make sense for all points in the compact set $[0,1]_{2}^{n}$. If Equation 9 is false then, by the analysis above, we can find some global maximum $A \in[0,1]_{2}^{n}$ such that $F(A)>0$. We will assume the existence of such an $A$ and derive a contradiction. We begin with an easy result:

Lemma 4.1 All coordinates of $A$ are positive, and 4 consecutive coordinates of $A$ cannot coincide

Proof: If some coordinate of $A$ is 0 then all coordinates of $A$ are 0 , since $A \in[0,1]_{2}^{n}$. But then $F(A)=0$, a contradiction. Hence all coordinates of $A$ are positive. We compute

$$
F_{c}(t, t, t, t, e)=\frac{t^{2}(t-e)}{(1+2 t)\left((1+e)(1+t)-t^{2}\right)} .
$$

If $e>t$ then $F_{c}<0$ and we can increase $F$ by decreasing $c$ and keeping $c>0$. If $e<t$ then $F_{c}>0$ and we can increase $F$ by increasing $c$ and keeping $c<1$. Hence $e=t$. Cycling through the coordinates, we see that they all coincide. But then $F(A)=0$, a contradiction.

Here is the main idea: After a huge amount of trial and error we discovered the following result:

Lemma 4.2 Let

$$
\eta(x, y)=\frac{2 \max \left(x^{2}, y^{2}\right)}{x+y}
$$

For any $U \in(0,4 / 3]$ let $\Omega_{U} \subset \boldsymbol{R}^{5}$ denote those points $(a, b, c, d, e)$ such that

- $-b<a \leq b \leq U \geq c \geq d \geq e>-d$.

- $\eta(a, b)=\eta(c, d)=\eta(d, e)=U$.

- If $b \geq c$ then $\eta(b, c) \leq U$.

We have $F_{c}(a, b, c, d, e) \leq 0$ for all $(a, b, c, d, e) \in \Omega_{U}$, with equality only if $a=b=c=d=e$. 
Given Lemma 4.2 we can finish the proof of Equation 9. If $(x, y) \in[0,1]^{2}$ satisfy $x / y \in[1 / 2,2]$ and $x \leq y$ then

$$
\eta(x, y)=y \eta\left(x y^{-1}, 1\right)=\frac{2 y}{1+x y^{-1}} \leq \frac{2}{1+x y^{-1}} \leq \frac{2}{1+1 / 2}=4 / 3
$$

The same bound holds when $y \leq x$, by symmetry. Therefore $\eta(x, y) \leq 4 / 3$ provided $(x, y) \in[0,1]^{2}$ and $x / y \in[1 / 2,2]$.

Using the dihedral symmetry of $F$ we can cycle and/or reverse the coordinates in $A$ so as to arrange that

$$
c \geq d ; \quad \max (\eta(a, b), \eta(b, c), \eta(d, e)) \leq \eta(c, d) \leq 4 / 3 ; \quad F_{c} \geq 0
$$

The condition $F_{c} \geq 0$ comes about as follows: If $F_{c}<0$ then we can increase $F$ by decreasing $c$ and keeping $c \leq 1$, contradicting the maximality of $A$.

Let $U=\eta(c, d) \in(0,4 / 3]$. We have

$$
U=\eta(c, d)=2 c^{2} /(c+d) \geq c .
$$

Hence $U \geq c$. If $b \leq c$ then $b \leq U$. If $b \geq c$ then $b \leq b^{2} /(b+c)=\eta(b, c) \leq U$, so again $b \leq U$. In short, we have

$$
0<b \leq U \geq c \geq d>0
$$

Lemma 4.3 Let $U>0$. Suppose $x \in(0, U]$ and

$$
y=\frac{2 x^{2}}{U}-x=\left(\frac{2 x}{U}-1\right) x
$$

Then $-x<y \leq x$ and $\eta(x, y)=U$.

Proof: Since $x \in(0, U]$ we get $-x<y \leq x$ by Equation 41. Then we have $\eta(x, y)=2 x^{2} /(x+y)=2 x^{2} /\left(2 x^{2} / U\right)=U$.

By Lemma 4.3 we can find $a^{\prime}$ and $e^{\prime}$ such that

$$
-b<a^{\prime} \leq b ; \quad \eta\left(a^{\prime}, b\right)=U ; \quad d \geq e^{\prime}>-d ; \quad \eta\left(d, e^{\prime}\right)=U .
$$

Equations 39, 40 and 42 combine to say that $\left(a^{\prime}, b, c, d, e^{\prime}\right) \in \Omega_{U}$. 
Lemma $4.4 a^{\prime} \leq a$ and $\eta(\alpha, b)<2$ provided that $\alpha \in\left[a^{\prime}, b\right]$. Likewise $e^{\prime} \leq e$ and $\eta(d, \epsilon)<2$ provided that $\epsilon \in\left[e^{\prime}, d\right]$.

Proof: We will prove the first statement. The second statement has the same proof. If $a>b$ then obviously $a^{\prime}<a$. If $a^{\prime} \in(-b, b]$ then again $a^{\prime} \leq a$ because $\eta(a, b) \leq \eta\left(a^{\prime}, b\right)$ and $\eta(x, b)=2 b^{2} /(x+b)$ is decreasing for $x \in(-b, b]$. In all cases, $a^{\prime} \leq a$. We just saw that $\eta(x, b)$ is monotone on $(-b, b]$. Hence $\eta(\alpha, b) \in\left[\eta(b, b), \eta\left(a^{\prime}, b\right)\right]=[b, U]$. Hence $\eta(\alpha, b) \leq 4 / 3<2$.

From Equation 35 we have

$$
F_{a c}(\alpha, b, c, *, *)=\frac{-b^{2}}{\left((1+\alpha)(1+c)-b^{2}\right)^{2}}
$$

Before finishing our proof we have to deal with the irritating possibility that the denominator in Equation 43 vanishes.

Lemma 4.5 Let $v>0$. Suppose, for $j=1,2$, that $u_{j}>-v$ and either $u_{j} \geq v$ or $\eta\left(u_{j}, v\right)<2$. Then $\left(1+u_{1}\right)\left(1+u_{2}\right)-v^{2}>0$.

Proof: If $u_{j}>v$ then $u_{j}>v^{2}-v$. If $u_{j} \in(-v, v]$ then $\eta\left(u_{j}, v\right)=2 v^{2} /\left(u_{j}+v\right)$ is monotone decreasing in $u_{j}$. Since $\eta\left(v^{2}-v, v\right)=2$ we have $u_{j}>v^{2}-v$. Thus $\left(1+u_{1}\right)\left(1+u_{2}\right)-v^{2}>\left(1+v^{2}-v\right)\left(1+v^{2}-v\right)-v^{2}=(v-1)^{2}\left(v^{2}+1\right) \geq 0$.

By Lemma 4.5 the denominator in Equation 43 is nonzero for any $\alpha>-b$ such that $\alpha \geq b$ or $\eta(\alpha, b)<2$. From the second statement of Lemma 4.4 we see that these conditions hold for all $\alpha \in\left[a^{\prime}, a\right]$. Hence $F_{c}(\alpha, b, c, *, *)$ is finite, negative, and uniformly bounded below for all $\alpha \in\left[a^{\prime}, a\right]$. Hence $F_{c}$ strictly increases as we decrease $\alpha$ from $a$ down to $a^{\prime}$. The same arguments work for $e$. Hence

$$
F_{c}=F_{c}(a, b, c, d, e) \leq F_{c}\left(a^{\prime}, b, c, d, e^{\prime}\right)
$$

with equality iff $a=a^{\prime}$ and $e=e^{\prime}$. Combining Equation 44, Equation 39, and Lemma 4.2 we get $F_{c}(a, b, c, d, e)=F_{c}\left(a^{\prime}, b, c, d, e^{\prime}\right)=0$. This forces $a=a^{\prime}=b=c=d=e^{\prime}=e$. In summary, at least 4 consecutive coordinates of $A$ coincide. This contradicts Lemma 4.1. So, Lemma 4.2 implies Equation 9 . 


\subsection{Lemma 4.2 Reduced to Lemma 4.9}

Lemma 4.2 boils Equation 9 (for any choice of $n$ ) down to a 3-dimensional algebra problem. To see this, we will produce a rational surjection

$$
S: \Upsilon=(0,1]^{2} \times[0,1) \quad \longrightarrow \quad \Omega=\bigcup_{U \in(0,4 / 3]} \Omega_{U}
$$

$S$ is actually a bijection, but we don't care about this.

Given $(X, Y, Z) \in \Upsilon$ define $S(X, Y, Z)=(a, b, c, d, e)$ as follows:

$$
\begin{gathered}
r=\frac{1+\sqrt{5}}{4} ; \quad U=\frac{4}{3} X ; \quad \widehat{b}=(1-Y) r U+Y U ; \quad b=\widehat{b}(1-Z) ; \\
a=\frac{2 b^{2}}{U}-b ; \quad c=\frac{2 \widehat{b}^{2}}{U}-\widehat{b} ; \quad d=\frac{2 c^{2}}{U}-c ; \quad e=\frac{2 d^{2}}{U}-d ; \quad
\end{gathered}
$$

Compare Equation 41. See also $§ 4.4$.

Lemma 4.6 $S(X, Y, Z) \subset \Omega_{U}$ for all $(X, Y, Z) \in \Upsilon$.

Proof: From equation 46 we have $0<b \leq \widehat{b} \leq U$. By Lemma 4.3 we have $a \leq b$ and $\eta(a, b)=U$. Since $\widehat{b} \leq U$ we get $U \geq c$ and $\eta(\widehat{b}, c)=U$ by Lemma 4.3. Using the file makepoly we find that $c=X \gamma / 3$ and $d=X Y \delta / 3$, where

$$
\begin{gathered}
\gamma=2+(-5+3 \sqrt{5}) Y+(7-3 \sqrt{5}) Y^{2} \\
\delta=(-5+3 \sqrt{5})+(42-18 \sqrt{5}) Y+(-80+36 \sqrt{5}) Y^{2}+(47-21 \sqrt{5}) Y^{3} .
\end{gathered}
$$

These two polynomials have positive coefficients. Hence $c, d>0$. Since $U \geq c$ we get $c \geq d$ and $\eta(c, d)=U$ by Lemma 4.3. Since $U \geq d$ we get $d \geq e$ and $\eta(d, e)=U$ by Lemma 4.3. All in all, $-b<a \leq b \leq U \geq c \geq d \geq e>-d$ and $\eta(a, b)=\eta(c, d)=\eta(d, e)=U$. Finally, if $b>c$ then $\eta(b, c) \leq U$. This follows from the fact that $\eta(\widehat{b}, c)=U$ and the fact that, when $x>c>0$, the function $\eta(x, c)=2 x^{2} /(c+x)$ is monotone increasing.

Lemma 4.7 Suppose $x \in(0, U]$ and $\eta(x, y)=U$ and $y \leq x$. Then $y$ satisfies Equation 41. Moreover, if $y>0$ then $x \in(U / 2, U]$.

Proof: The first statement comes directly from Solving Equation 37 for $y$ in terms of $x$ and $U$. The second statement is immediate from Equation 41. 
Lemma 4.8 $S$ maps $\Upsilon$ onto $\Omega$.

Proof: We have $S(X, 0, Z)=(*, *, U / 2,0, *)$ and $S(X, 1, Z)=(*, *, U, U, *)$ by Equation 47 . Thus $c$ sweeps out $(U / 2, U]$ and $d$ sweeps out $(0, U]$ as $Y$ ranges in $(0,1]$. Furthermore, $d$ determines $e$ by Equation 46. By Lemma 4.7 we get all possible $(*, *, c, d, e) \in \Omega_{U}$ by varying $Y$ in $(0,1]$. If $Y$ is fixed, $b$ sweeps out $(0, \widehat{b}]$ as $Z$ ranges in $[0,1)$. From the analysis at the end of Lemma 4.6 we see that $b$ attains every value such that either $b \leq c$ or else $b \geq c$ and $\eta(b, c) \leq U$. Finally, $b$ determines $a$. Thus by first adjusting $Y$, and then adjusting $Z$, we reach all points in $\Omega_{U}$. Finally, by varying $X \in(0,1]$ we get all of $\Omega$ in the image.

Let $(a, b, c, d, e)=S(X, Y, Z)$. Using the file makepoly we compute that

$$
F_{c}(a, b, c, d, e)=X^{2} \frac{P(X, Y, Z)}{Q(X, Y, Z)} .
$$

Here $P$ and $Q$ are polynomials such that $Q(1 / 2,1 / 2,1 / 2)<0$. See the routine ComputePolynomials [] in $\S 4.4$. Below we prove

Lemma 4.9 $P(X, Y, Z) \geq 0$ on $\Upsilon$, with equality only if $Y=1$ and $Z=0$.

Corollary 4.10 $Q(X, Y, Z)<0$ for all $(X, Y, Z) \in(0,1)^{3}$.

Proof: By Lemma 4.5, the denominators in Equation 34 never vanish when $(a, b, c, d, e) \in \Omega$. Hence $P(X, Y, Z) / Q(X, Y, Z)<\infty$ for all $(X, Y, Z) \in$ $(0,1)^{3}$. Since $P(X, Y, Z)>0$ for all $(X, Y, Z) \in(0,1)^{3}$ we have $Q(X, Y, Z) \neq$ 0 , so the sign of $Q$ never changes on $(0,1)^{3}$. Finally, $Q(1 / 2,1 / 2,1 / 2)<0$.

Lemma 4.9, Corollary 4.10, and Equation 48 imply that $F_{c}(a, b, c, d, e) \leq 0$ for all $(a, b, c, d, e) \in \Omega$. If $F_{c}(a, b, c, d, e)=0$ then $P(X, Y, Z)=0$. But then $Z=0$ and $Y=1$. Hence $c=U$. Then $d=U$ by Equation 41. Similarly $\widehat{b}=c=U$. Since $Z=0$ we have $b=\widehat{b}$. Since $b=U$ and $\eta(a, b)=U$ we get $a=U$. Similarly $e=U$. In short, $a=b=c=d=e$.

To finish the proof of Equation 9 we just have to establish Lemma 4.9. 


\subsection{Proof of Lemma 4.9}

Given $I=\left(i_{1}, \ldots, i_{k}\right) \in(\boldsymbol{N} \cup\{0\})^{k}$ we let $X^{I}=X_{1}^{i_{1}} \ldots X_{k}^{i_{k}}$. Any polynomial $F \in \boldsymbol{R}\left[X_{1}, \ldots, X_{k}\right]$ can be written succinctly as $F=\sum A_{I} X^{I}$ with $A_{I} \in \boldsymbol{R}$. If $I^{\prime}=\left(i_{1}^{\prime}, \ldots, i_{k}^{\prime}\right)$ we write $I^{\prime} \leq I$ if $i_{j}^{\prime} \leq i_{j}$ for all $j=1, \ldots, k$. We call $F$ positive dominant if $\sum_{I^{\prime} \leq I} A_{I^{\prime}}>0$ for all $I$. When $k=0$, positive dominant means a positive number.

Lemma 4.11 If $F$ is positive dominant then $F$ is positive on $[0,1]^{k}$.

Proof: Write $F=f_{0}+f_{1} X_{k}+\ldots+f_{m} X_{k}^{m}$, where $f_{j} \in \boldsymbol{R}\left[X_{1}, \ldots, X_{k-1}\right]$. Let $F_{j}=f_{0}+\ldots+f_{j}$. The positive dominance of $F$ implies the positive dominance of $F_{j}$ for all $j$. By induction, $F_{j}>0$ on $[0,1]^{k-1}$. When $X_{k}=0$, we have $F=F_{0}>0$ on $\{0\} \times[0,1]^{k-1}$. Suppose $X_{k} \in(0,1]$. Since $X_{k}^{i} \geq X_{k}^{j}$ for $i<j$,

$$
\begin{gathered}
F=f_{0}+f_{1} X_{k}+\ldots+f_{m} X_{k}^{m} \geq F_{1} X_{k}+f_{2} X_{k}^{2}+\ldots+f_{m} X_{k}^{m} \geq \\
F_{2} X_{k}^{2}+f_{3} X_{k}^{3}+\ldots+f_{m} X_{k}^{m} \geq \ldots \geq F_{m} X_{k}^{m}>0 . \boldsymbol{\phi}
\end{gathered}
$$

In the file makepoly we compute that

$$
P=P_{0}+P_{1} Z+P_{2} Z^{2}+P_{3} Z^{3} ; \quad P_{0}=(1-Y) \widehat{P}_{0} ; \quad P_{3}=X \widehat{P}_{3} .
$$

Here $P_{0}, \widehat{P}_{0}, P_{1}, P_{2}, P_{3}, \widehat{P}_{3} \in \boldsymbol{Z}[\sqrt{5}](X, Y)$. Now $P_{0}, P_{2}, P_{3}$ are not positive dominant, and $P_{2}$ is sometimes negative on $(0,1)^{2}$. We compute that

$$
\widehat{P}_{0} ; \quad P_{1} ; \quad \widehat{P}_{3} ; \quad \Pi_{1}=P_{0}+P_{1}+\frac{1}{2} P_{2} ; \quad \Pi_{2}=P_{0}+P_{1}+P_{2}+\frac{1}{2} P_{3}
$$

are all positive dominant. By Lemma 4.11 and Equation $49, P_{1}, \Pi_{1}, \Pi_{2}>0$ on $[0,1]^{2}$, and $P_{0}>0$ on $[0,1] \times[0,1)$, and $P_{3}>0$ on $(0,1] \times[0,1]$.

First we show that $P>0$ on $(0,1]^{2} \times(0,1)$. If $Z \in[1 / 2,1)$ then

$$
P / Z^{2}=P_{0} / Z^{2}+P_{1} / Z+P_{2}+Z P_{3} \geq P_{0}+P_{1}+P_{2}+\frac{1}{2} P_{3}=\Pi_{2}>0 .
$$

If $Z \in(0,1 / 2]$ then the nontrivial case occurs when $P_{2}(X, Y, Z) \leq 0$. In this case we have $Z^{2} P_{2}(X, Y, Z) \geq(Z / 2) P_{2}(Z, Y, Z)$, which yields

$P_{0}+P_{1} Z+P_{2} Z^{2}+Z^{3} P_{3}>Z P_{0}+Z P_{1}+Z^{2} P_{2} \geq Z\left(P_{0}+P_{1}+\frac{1}{2} P_{2}\right)=Z \Pi_{1}>0$.

If $P(X, Y, Z)=0$ for some $(X, Y, Z) \in \Upsilon$ then $Z=0$. But then we have $P(Z, Y, 0)=P_{0}(X, Y)=0$, which forces $Y=1$. This completes the proof. 


\subsection{Some Mathematica Code}

The code here is copied from our file makepoly.

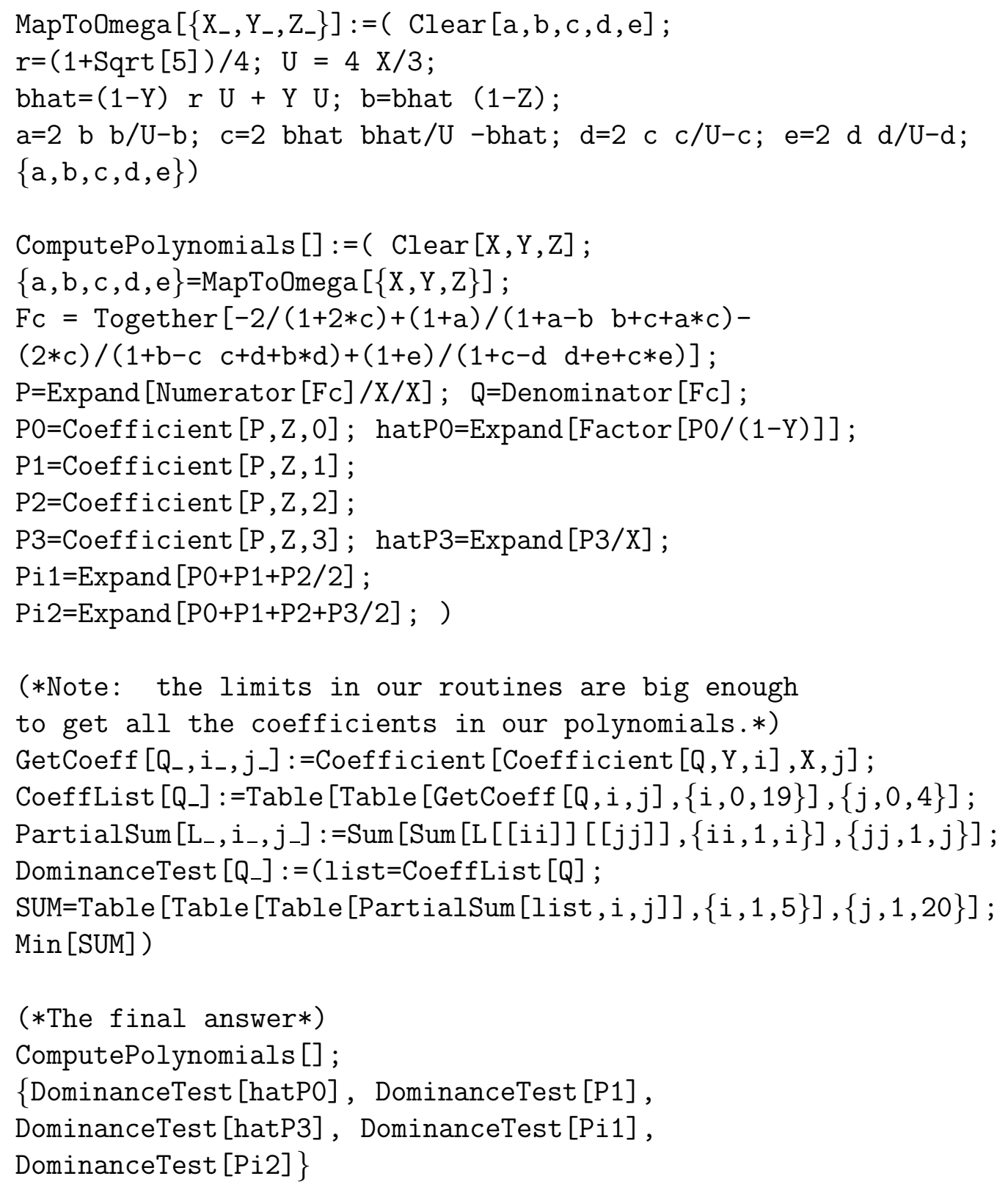




\section{$5 \quad$ Proof of Theorem 1.1 and Corollary 1.2}

\subsection{A Compactness Result}

We fix $n \geq 5$ for this entire chapter. Let $\phi$ be the energy function from Equation 4 . Let $W \subset S^{1}$ be an $n$-gon, as in $\S 1$. We begin this chapter with two short technical results, which will be used below.

Lemma 5.1 Let $Z=\left(z_{1}, \ldots, z_{n}\right)$ be the list of cross ratios for an $n$-gon $W$ in $S^{1}$, with $n \geq 5$. Then $z_{j}+z_{j+1}<1$ for all $j$.

Proof: We write $W=\left(w_{1}, \ldots, w_{n}\right)$. We will show that $z_{1}+z_{2}<1$, the general case being the same. An easy computation shows that

$$
\frac{z_{1}}{1-z_{2}}=\frac{\chi\left(w_{1}, w_{2}, w_{3}, w_{4}\right)}{1-\chi\left(w_{2}, w_{3}, w_{4}, w_{5}\right)}=\chi\left(w_{1}, w_{2}, w_{3}, w_{5}\right)<1 .
$$

The last inequality comes from the fact that the points $w_{1}, w_{2}, w_{3}, w_{5}$ appear in cyclic order on $S^{1}$. This lemma is immediate from Equation 51.

Let $\mathcal{Y}$ denote those $n$-gons such that $w_{1}, w_{2}, w_{3}$ are consecutive vertices of a regular $n$-gon, with $w_{1}=1$. Then $w_{2}=\exp (2 \pi i / n)$ and $w_{3}=\exp (4 \pi i / n)$. Every $n$-gon is conformally equivalent to an element of $\mathcal{Y}$. The space $\mathcal{Y}$ is homeomorphic to $(0,1)^{n-3}$. To see this note that there is an interval of choices for $w_{4}$. Once $w_{4}$ is chosen, there is an interval of choices for $w_{5}$. And so forth. The only conformally regular element of $\mathcal{Y}$ is the regular $n$-gon. The energy function $\phi$ gives us a map $\phi: \mathcal{Y} \rightarrow(0,1)$.

Lemma 5.2 If $\left\{W_{k}\right\}$ is a sequence in $\mathcal{Y}$ which exits every compact subset of $\mathcal{Y}$ then $\phi\left(W_{k}\right) \rightarrow 0$.

Proof: Let $z_{k 1}, \ldots, z_{k n}$ be the set of cross ratio invariants for $W_{k}$. Since $z_{k j} \in(0,1)$ for all indices we have $\phi\left(W_{k}\right)<\min _{j}\left(z_{k j}\right)$. If $\left\{W_{k}\right\}$ exits every compact subset of $\mathcal{Y}$ then by passing to subsequence we arrange that some cross ratio $z_{k j}$ either tends to 0 or to 1 . In the latter case, $z_{k, j+1}$ tends to 0 by Lemma 5.1. Hence, in all cases, $\min _{j} z_{k j} \rightarrow 0$. Hence $\phi\left(W_{k}\right) \rightarrow 0$. 


\subsection{The Convergence of Cross Ratios}

Combining Equations 6, 8 and 9 we get Equation 5: $\phi\left(W^{\prime}\right) \geq \phi(W)$ for any $n$-gon $W \subset S^{1}$, with equality iff $W$ is conformally regular.

Lemma 5.3 Let $W^{\prime}, W^{(2)}, W^{(3)}, \ldots$ be as in $\S 1$. Let $Z_{k}=\left(z_{k 1}, \ldots, z_{k n}\right)$ be the list of cross ratios of $W^{(k)}$. Then $\left\{Z_{k}\right\}$ converges to the sequence of cross ratios associated to the regular $n$-gon.

Proof: Let $A_{k}=\left(a_{k 1}, \ldots, a_{k n}\right)$ be such that $a_{k j}=\sqrt{z_{k j}}$. Let $\phi_{k}=\phi\left(W^{(k)}\right)$. Then $\left\{\phi_{k}\right\}$ is a monotone increasing sequence, bounded above by 1 . Thus $\phi_{k}$ converges to some $\phi_{\infty}$. Hence $\phi_{k+1} / \phi_{k} \rightarrow 1$. Let $Z_{\infty}$ be any subsequential limit of $\left\{Z_{n}\right\}$. Let $A_{\infty}$ be the corresponding subsequential limit of $\left\{A_{n}\right\}$. Both Equations 8 and 9 hold for $A_{\infty}$. Since $\phi_{k+1} / \phi_{k} \rightarrow 1$ we must have equality in both Equations 8 and 9 when they are applied to $A_{\infty}$. But this is only possible if $A_{\infty}$ is a constant sequence. But then $Z_{\infty}$ is a constant sequence. By Lemma 5.2 there is a compact subset of $\mathcal{Y}$ which contains some conformal image $V_{k}$ of $W^{(k)}$ for each $k$. By compactness, $Z_{\infty}$ is actually a list of cross ratios for an $n$-gon. Since $Z_{\infty}$ is a constant sequence, $Z_{\infty}$ must be the list of cross ratios of the regular $n$-gon. Hence all subsequential limits of $\left\{Z_{k}\right\}$ coincide and $\left\{Z_{k}\right\}$ converges to $Z_{\infty}$.

Corollary 5.4 There is a sequence of conformal transformations $\left\{T_{k}\right\}$ such that $T_{k}\left(W^{(k)}\right)$ converges to the regular $n$-gon.

Proof: Let $T_{k}$ be the conformal map such that $V_{k}=T_{k}\left(W^{(k)}\right) \in \mathcal{Y}$. Since the list of cross ratios of $V_{k}$ converges to the list for the regular $n$-gon, and the first three vertices of $V_{k}$ coincide with the first three vertices of the regular $n$-gon, it follows by induction on $n$ that the remaining vertices of $V_{k}$ converge to the remaining vertices of the regular $n$-gon as well.

Proof of Corollary 1.2: Since $\phi(W)$ only depends on the conformal equivalence class of $W$ it suffices, for our proof of Corollary 1.2, to prove that the restriction of $\phi$ to $\mathcal{Y}$ is maximized precisely at the regular element. By Lemma 5.2 , the function $\phi$ attains its global maximum for some polygon $W$. If $W \in \mathcal{Y}$ is not regular then $W$ is not conformally regular and $\phi\left(W^{\prime}\right)>\phi(W)$. This is a contradiction. Hence $W$ is regular. 


\subsection{An Embedding Result}

Before we finish our proof of Theorem 1.1 we need more technical results.

Say that a proper submanifold of $(0,1)^{n}$ is a subset of the form $f(U)$, where $U$ is an open subset of $\boldsymbol{R}^{k}$ and $f: U \rightarrow(0,1)^{n}$ is a smooth nonsingular map such that $f: U \rightarrow f(U)$ is a homeomorphism. Here $f(U)$ is equipped with the subspace topology.

Let $\mathcal{Z} \subset(0,1)^{n}$ denote those sequences $\left(z_{1}, \ldots, z_{n}\right)$ which arise as cross ratios of $n$-gons in $S^{1}$. Let $Z_{\infty}$ be the list of cross ratios of the regular $n$-gon.

Lemma $5.5 \mathcal{Z}$ is a proper submanifold of $(0,1)^{n}$. Moreover the tangent space to $\mathcal{Z}$ at $Z_{\infty}$ is perpendicular to the vector $(1, \ldots, 1)$.

Proof: Let $\mathcal{Y}$ be as in $\S 5.1$. Then $\mathcal{Y}$ is a smooth manifold, diffeomorphic to $(0,1)^{n-3}$, as we already mentioned. Every $n$-gon is equivalent to a unique $n$-gon in $\mathcal{Y}$, so the cross ratio map gives a smooth surjective map $f_{1}: \mathcal{Y} \rightarrow \mathcal{Z}$. On the other hand, the positions of $w_{2}, w_{3}, \ldots, w_{n-2}$ are determined by, and depend smoothly on, the cross ratios $\left(z_{1}, \ldots, z_{n}\right)$. Hence $f_{1}$ has a smooth inverse, $f_{2}$. The compositions $f_{1} \circ f_{2}$ and $f_{2} \circ f_{1}$ are both the identity maps, so that $d f_{1}$ is everywhere nonsingular, continuous, and injective.

It remains to show that $f_{1}$ is a homeomorphism onto its image. The map $f_{2}$ is continuous with respect to the subspace topology. In other words, if $Z_{1}, Z_{2} \subset \mathcal{Z}$ are close in terms of the ambient Euclidean distance on $(0,1)^{n}$, then $f_{2}\left(Z_{1}\right)$ and $f_{2}\left(Z_{2}\right)$ are close as polygons. Given all the other properties above, the kind of continuity enjoyed by $f_{2}$ implies that $f_{1}$ is a homeomorphism onto its image. Hence $\mathcal{Z}$ is a proper submanifold.

By symmetry, the tangent space to $\mathcal{Z}$ at $Z_{\infty}$ either contains $(1, \ldots, 1)$ or is perpendicular to $(1, \ldots, 1)$. The fact that $\phi$, restricted to $\mathcal{Z}$, is maximized at $Z_{\infty}$ rules out the former possibility: Otherwise we could increase $\phi$, while staying in $\mathcal{Z}$, by moving in the direction of $(1, \ldots, 1)$.

Corollary 5.6 Let $\left\{Z_{k}\right\} \in \mathcal{Z}$ be a sequence of points which converges to $Z_{\infty}$. Let $\widehat{\epsilon}_{k}$ denote the distance from $Z_{k}$ to the diagonal $\Delta$. Then $\left\|Z_{k}-Z_{\infty}\right\|<2 \widehat{\epsilon}_{k}$ for $k$ sufficiently large.

Proof: This follows from the fact that $\mathcal{Z}$ is a proper submanifold, perpendicular to $\Delta$ at $Z_{\infty}$, and from the fact that the tangent space to $\mathcal{Z}$ at $Z_{\infty}$ approximates $\mathcal{Z}$ to first order. 


\subsection{The End of the Proof}

Let $Z_{k}, A_{k}$, etc. be as in Lemma 5.3. Recall that $\phi_{k}=\prod_{j} z_{k j}$. We define $\epsilon_{k}=\phi_{\infty}-\phi_{k}$. By Corollary 1.2 we have $\epsilon_{k} \geq 0$ for all $k$. Let $\widehat{\epsilon}_{k}$ be as in Corollary 5.6. Below, $C_{1}, C_{2}, C_{3}, C_{4}$ are positive constants which depend on the initial $n$-gon $W$.

Lemma $5.7\left(\widehat{\epsilon}_{k}\right)^{2} \geq C_{1} \epsilon_{k}$. for $k$ sufficiently large.

Proof: Let $\mathcal{Z}$ be as in Lemma 5.5. We have $Z_{k} \in \mathcal{Z}$. By Corollary 5.6 we have $\left\|Z_{k}-Z_{\infty}\right\|<2 \widehat{\epsilon}_{k}$ once $\widehat{\epsilon}_{k}$ is sufficiently small. We can interpret $\phi$ as a map from $(0,1)^{n} \rightarrow(0,1)$. Namely, we have $\phi\left(z_{1}, \ldots, z_{n}\right)=\prod z_{j}$. Obviously $\phi$ is smooth. The gradient $\nabla \phi$, evaluated at $Z_{\infty}$, is parallel to $(1, \ldots, 1)$. Hence $\mathcal{Z}$ is perpendicular to $\nabla \phi$ at $Z_{\infty}$. From Taylor's theorem applied to $\phi$, we get

$$
\epsilon_{k}=\phi\left(Z_{\infty}\right)-\phi\left(Z_{k}\right) \leq \frac{1}{4} C_{1}^{-1}\left\|Z_{\infty}-Z_{k}\right\|^{2} \leq C_{1}^{-1}\left(\widehat{\epsilon}_{k}\right)^{2}
$$

provided $k$ is large.

Lemma $5.8 \epsilon_{k}-\epsilon_{k+1} \geq C_{2}\left(\widehat{\epsilon}_{k}\right)^{2}$ for $k$ sufficiently large.

Proof: Let $A=A_{\infty}=\left(a_{\infty}, \ldots, a_{\infty}\right)$, as in Lemma 5.3. Let $q_{k}=\left(a_{k 1}, \ldots, a_{k n}\right)$, with $a_{k j}=\sqrt{z_{k j}}$. Since $q_{k} \rightarrow A$ we can apply Lemma 3.1 for $k$ large enough. The map $f(x)=\sqrt{x}$ is bi-Lipschitz in a neighborhood of $z_{\infty}=a_{\infty}^{2}$ and hence $\delta\left(q_{k}\right) \geq C_{3} \widehat{\epsilon}_{k}$. By Lemma 3.1 we have $E\left(q_{k}\right) \geq C_{4}\left(\widehat{\epsilon}_{k}\right)^{2}$. From Equations 28 and 33 , and the definitions, we have

$$
\left.\log \phi_{k+1}-\log \phi_{k}=2 E\left(q_{k}\right)-2 F\left(q_{k}\right) \geq^{*} 2 E\left(q_{k}\right)\right) \geq 2 C_{4}\left(\widehat{\epsilon}_{k}\right)^{2} .
$$

The starred inequality comes from the fact that $F \leq 0$ on $(0,1)^{n}$ by Equation 9. Since the function log is bi-Lipschitz in a neighborhood of $\phi_{\infty}$ we get that $\phi_{k+1}-\phi_{k} \geq C_{2}\left(\widehat{\epsilon}_{k}\right)^{2}$ for $k$ large enough. But $\phi_{k+1}-\phi_{k}=\epsilon_{k}-\epsilon_{k+1}$.

By Lemmas 5.7 and 5.8 we have

$$
\epsilon_{k}-\epsilon_{k+1} \geq C_{1} C_{2} \epsilon_{k}
$$


for $k$ sufficiently large. Since $\epsilon_{j} \geq 0$ for all $j$ we have $C_{1} C_{2} \leq 1$. Setting $D=1-C_{1} C_{2} \in[0,1)$, we have

$$
\epsilon_{k+1} \leq D \epsilon_{k} ; \quad D \in[0,1)
$$

Since this holds true for all $k$ sufficiently large we see that $\epsilon_{k}$ decays exponentially. Lemma 5.8 now says that $\widehat{\epsilon}_{k}$ decays exponentially. Corollary 5.6 gives $\left\|Z_{k}-Z_{\infty}\right\|<2 \widehat{\epsilon}_{k}$ for large $k$. Hence $\left\|Z_{k}-Z_{\infty}\right\|$ also decays exponentially. The points of $V_{k}$ are smooth functions of the coordinates in $Z_{k}$. Since $\left\|Z_{k}-Z_{\infty}\right\|$ decays exponentially, $V_{k}$ converges exponentially fast to the regular $n$-gon $V_{\infty}$.

For the moment we fix $k$. Let $\left(v_{1}^{\prime \prime}, \ldots, v_{n}^{\prime \prime}\right)$ be the successive points of $V_{k-2}^{\prime \prime}$ labelled so that $\left(v_{1}^{\prime \prime}, v_{2}^{\prime \prime}, v_{3}^{\prime \prime}\right)$ are, respectively, close to $\left(v_{1}, v_{2}, v_{3}\right)$, the first three points of $V_{\infty}$. The points of $V_{k-2}^{\prime \prime}$ depend smoothly on the points of $V_{k-2}$, which converge exponentially fast to the points of the regular $n$-gon. Hence $v_{j}^{\prime \prime}$ converges exponentially fast to $v_{j}$ for $j=1,2,3$.

For any conformal transformation $T$ let $|T|$ denote the maximum Euclidean distance $T$ moves a point on $S^{1}$. Let $T_{k}$ be as in Corollary 5.4. Let $G_{k}=T_{k} T_{k-2}^{-1}$. For $k \geq 1$ we have

$$
T_{2 k}=G_{2 k} \circ G_{2 k-2} \circ \ldots \circ G_{2} .
$$

We compute

$$
\begin{gathered}
G_{k}\left(V_{k-2}^{\prime \prime}\right)=T_{k} T_{k-2}^{-1}\left(V_{k-2}^{\prime \prime}\right)={ }^{*} T_{k}\left(\left(T_{k-2}^{-1}\left(V_{k-2}\right)\right)^{\prime \prime}\right)= \\
T_{k}\left(\left(W^{(k-2)}\right)^{\prime \prime}\right)=T_{k}\left(W^{(k)}\right)=V_{k} .
\end{gathered}
$$

The starred equality comes from the naturality of the conformal averaging process. Equation 57 says, in short, that $G_{k}\left(V_{k-2}^{\prime \prime}\right)=V_{k}$. A conformal transformation is determined by its action on 3 points. Hence $G_{k}$ is determined by the action $\left(v_{1}^{\prime \prime}, v_{2}^{\prime \prime}, v_{3}^{\prime \prime}\right) \rightarrow\left(v_{1}, v_{2}, v_{3}\right)$. Given that $v_{j}^{\prime \prime}$ converges exponentially fast to $v_{j}$, for $j=1,2,3$, and the points $v_{1}, v_{2}, v_{3}$ are distinct and independent of $k$, we see that $\left|G_{k}\right|$ decays exponentially.

Since $\left|G_{k}\right|$ decays exponentially, the maps $T_{2 k}$ converge exponentially fast to a conformal transformation $T_{\infty}$. See Equation 56. As above, we know that $V_{k} \rightarrow V_{\infty}$ exponentially fast. Therefore, $W^{(2 k)}=T_{2 k}^{-1}\left(V_{2 k}\right)$ converges exponentially fast to $W^{(\infty)}=T_{\infty}^{-1}\left(V_{\infty}\right)$. This completes the proof of Theorem 1.1. 


\section{Proof of Theorems 1.3, 1.4, 1.5 and 1.6}

\subsection{Small Conformal Circles and Theorem 1.3}

$[\mathbf{K}]$ has the classification of conformal circles. Here is the list of small ones.

- The parabolic circle: $\boldsymbol{R} / \boldsymbol{Z}$ is a small conformal circle. The canonical Riemannian metric on $\boldsymbol{R} / \boldsymbol{Z}$ is, of course, given by $d x$.

- The elliptic circles: Let $S \subset S^{1}$ be a proper arc. Let $I: S^{1} \rightarrow S^{1}$ be an isometric rotation which identifies the endpoint of $S$. Then $S / I$ is a conformal circle. Arc-length $d s$ on $S^{1}$ gives the canonical Riemannian metric on $S / I$. These circles are parametrized by their length.

- The hyperbolic circles: Let $T_{\lambda}$ be the map $x \rightarrow \lambda x$, with $\lambda>1$. Then $(0, \infty) / T_{\lambda}$ is a small conformal circle. The metric $d x / x$ on $(0, \infty)$ is preserved by $T_{\lambda}$ and gives a canonical Riemannian metric on $(0, \infty) / T_{\lambda}$. These circles are parametrized by their length, $\log (\lambda)$.

Proof of Theorem 1.3: Let $S$ be a small conformal circle. The proof of Lemma 5.3 is the same here. The proof of Corollary 5.4 goes through word for word, once we redefine the space $\mathcal{Y}$ to be the set of $n$-gons $W=\left(w_{1}, \ldots, w_{n}\right)$ where $w_{1}$ is some pre-chosen origin in $S$. Every automorphism of a small conformal circle is a Riemannian isometry, so every $n$-gon is conformally equivalent to a unique $n$-gon in $\mathcal{Y}$. Lemma 5.1 goes through, because the local developing maps are injective.

It is worth explaining the details of Lemma 5.2. Suppose $\left\{W_{k}\right\}$ exits every compact subset of $\mathcal{Y}$. Let $I_{k j}$ be the interval between $w_{k j}$ and $w_{k, j+1}$. Here $\left\{w_{k 1}, \ldots, w_{k n}\right\}$ are the successive points of $W_{k}$. After relabelling we can arrange that $I_{k 2}$ shrinks to a point but $I_{k 3}$ does not. Let $\mathbf{d e v}_{k}=\mathbf{d e v}_{w_{k 1}}$. We can choose $\mathbf{d e v}_{k}$ to lie within a compact set of maps. By compactness, both $U_{k}=\operatorname{dev}_{k}\left(I_{k 3}\right)$ and $V_{k}=S^{1}-\operatorname{dev}_{k}\left(S-w_{k 1}\right)$ have length uniformly bounded away from 0 . For $j=2,3,4,5$ let $s_{k j}=\operatorname{dev}_{k}\left(w_{k j}\right)$. Then $s_{k 2}$ and $s_{k 3}$ converge to a single point in $S^{1}$ whereas $s_{k 4}$ and $s_{k 5}$ are separated from $s_{k 2}$ and $s_{k 3}$ on one side by the large arc $U_{k}$ and on the other by the large arc $V_{k}$. This shows that $\chi\left(w_{k 2}, w_{k 3}, w_{k 4}, w_{k 5}\right) \rightarrow 0$. Hence $\phi\left(W_{k}\right) \rightarrow 0$.

The rest of $\S 5$ goes through pretty much word for word. 


\subsection{Medium Conformal Circles and Theorem 1.4}

Here we describe $S_{-}^{1}$. Let $X=\boldsymbol{R} \cup \infty$. Let $\widetilde{X}$ denote the universal cover of $X$. Let $p: \widetilde{X} \rightarrow X$ denote the projection map. Let $(0,0) \in \widetilde{X}$ be a lift of 0 and let $\boldsymbol{R}_{0} \subset \widetilde{X}$ be the lift of $\boldsymbol{R}$ which contains $(0,0)$. Let $(x, 0)=p^{-1}(x) \in \boldsymbol{R}_{0}$.

Let $G$ be the deck transformation group of $\widetilde{X}$. Then $G$ is isomorphic to $\boldsymbol{Z}$, the group of integers. Of the two generators of $G$ let $T$ be the generator which has the following property: The two sequences $\{(n, 0)\}$ and $\{T(-n, 0)\}$ converge to the same point of $\widetilde{X}$ as $n \rightarrow+\infty$.

Let $P_{-}$be the parabolic automorphism of $\boldsymbol{R} \cup \infty$ given the equation $P_{-}(x)=x-1$ and $P_{-}(\infty)=\infty$. Let $\widetilde{P}_{-}$be the lift of $P_{-}$to $\widetilde{X}$, such that

$$
\widetilde{P}_{-}(x, 0)=(x-1,1) .
$$

Equation 58 is enough to determine the action of $\widetilde{P}$ on all of $\widetilde{X}$. Then $S_{-}^{1}$ is the quotient $\widetilde{X} / P_{-}$. The distintuished point of $S_{-}^{1}$ is $\infty$.

Note that $\mathbf{d e v}_{\infty}$ maps $S_{-}^{1}-\infty$ onto $\boldsymbol{R}$. If $x \in S_{-}^{1}$ is any other point then $\operatorname{dev}_{x}$ maps $S_{-}^{1}-\{x\}$ onto $(x \cup \infty) \cup \infty \cup(-\infty, x-1)$, which is a proper arc of $S^{1}=\boldsymbol{R} \cup \infty$. This shows that $S_{-}^{1}$ is a medium conformal circle.

It is worth pointing out that Lemma 5.2 is false for $S_{-}^{1}$. The proof given in the previous section relies crucially on the local developing maps omitting more than one point, and there is no replacement proof. Indeed, Theorem 1.4 implies that Lemma 5.2 is false.

Proof of Theorem 1.4: The same argument as in Lemma 5.3 shows that the list $Z_{k}$ of cross ratios of $W^{(k)}$ converges to a constant list $Z_{\infty}$. This constant list lies in the closure of lists which correspond to actual $n$-gons in $S_{-}^{1}$. There is only one constant list with this property, and this is the list of cross ratios associated to the regular $n$-gon in $S^{1}$. Hence $Z_{\infty}$ must be the list of cross ratios of the regular $n$-gon. This is half of Theorem 1.4.

For the other half, suppose that $\left\{W^{(k)}\right\}$ does not exit every compact subset of the space $\mathcal{W}\left(S_{-}^{1}, n\right)$. Then some subsequence of $\left\{W^{(k)}\right\}$ converges to some $n$-gon $W_{\infty}$ of $S_{-}^{1}$. But the list of cross ratios of $W_{\infty}$ is the same as the list for conformally regular $n$-gons. But no $n$-gon in $S_{-}^{1}$ has the same list of cross ratios as an $n$-gon in $S^{1}$; otherwise we could work three points at a time and produce a conformal isomorphism between $S^{1}$ and $S_{-}^{1}$. 


\subsection{Large Conformal Circles and Theorem 1.5}

To see what can go wrong with the conformal averaging process for large conformal circles we consider an example. Let $S_{4}^{1}$ be the 4 -fold cover of $S^{1}$. If $w_{1}, w_{2}, w_{3}, w_{4} \in S_{4}^{1}$ all project to the same point in $S^{1}$ then there is no way to make a conformally natural assignment of a point $w_{*} \in\left(w_{2}, w_{3}\right)$. The problem is that there is a conformal automorphism of $S_{4}^{1}$ which fixes each $w_{j}$ but moves any other point. Thus, the conformal averaging process can break down on large conformal circles.

If $S$ is a large conformal circle, we say that an $n$-gon $W=\left(w_{1}, \ldots, w_{n}\right) \subset S$, with $n \geq 5$ is tame if, for every 4 consecutive points $w_{j}, w_{j+1}, w_{j+2}, w_{j+3}$, there is another point $x \in S-\left(w_{j}, w_{j+3}\right)$ such that $\operatorname{dev}_{x}\left(\left(w_{j}, w_{j+3}\right)\right)$ is contained in a proper arc of $S^{1}$. Here $\left(w_{j}, w_{j+3}\right)$ is the arc of $S$ bounded by $w_{j}$ and $w_{j+3}$ which contains $w_{j+1}$ and $w_{j+2}$. If $W$ is tame then the conformal average $W^{\prime}$ is defined and also the list of cross ratios of $W$ is defined. However, $W^{\prime}$ need not be tame. The conformal averaging process can be started for any tame $n$-gon, but it might break down eventually.

Let $S_{\theta}^{1}$ be as in the introduction. This space has a natural Riemannian metric, inherited from the round metric on $S^{1}$. The tameness condition translates into the statement that every 4 consecutive points of $W$ lie in an arc of $S_{\theta}^{1}$ of length less than $2 \pi$. For a regular $n$-gon on $S_{\theta}^{1}$ to have this property we must have $n>4 \theta$. We discuss this more in the next section.

Proof of Theorem 1.5: Let $W_{0} \in \mathcal{W}\left(S_{\theta}^{1}, n\right)$ be the regular $n$-gon. Suppose $W \in \mathcal{W}\left(S_{\theta}^{1}, n\right)$ is excessive: $\phi(W)>\phi\left(W_{0}\right)$. Let $Z_{0}=\left(z_{01}, \ldots, z_{0 n}\right)$ be the list of cross ratios of $W$. Let $(\zeta, \ldots, \zeta)$ be the list of cross ratios of $W_{0}$. We will suppose that $W^{(k)}$ is defined for all $k$ and derive a contradiction. If $W^{(k)}$ is defined for all $k$ then the list $Z_{k}$ of cross ratios of $W^{(k)}$ must converge to some constant list $Z_{\infty}=\left(\zeta^{\prime}, \ldots, \zeta^{\prime}\right)$, as in Lemma 5.3. Given that $\phi\left(W^{\infty}\right) \geq \phi(\underline{W})>\phi\left(W_{0}\right)$ we must have $\zeta^{\prime}>\zeta$.

Now, let $\widetilde{W}^{(k)}$ denote a lift of $W^{(k)}$ to $\widetilde{S}^{1}$. Imitating the proof of Corollary 5.4 , we can find a sequence $\left\{\widetilde{T}_{k}\right\}$ of conformal automorphisms of $\widetilde{S}^{1}$ such that $\widetilde{V}_{k}:=\widetilde{T}_{k}\left(\widetilde{W}^{(k)}\right)$ converges to an infinite collection $\widetilde{V}_{\infty}$ of evenly spaced points in $\widetilde{S}^{1}$. Every $n$ consecutive points of $\widetilde{V}_{k}$ lie in an arc of $\widetilde{S}^{1}$ having length precisely $2 \pi \theta$. By continuity, the same goes for every $n$ consecutive points of $\widetilde{V}_{\infty}$. But then the even spacing forces $\widetilde{V}_{\infty}$ to be a lift of $W_{0}$. Hence $\zeta^{\prime}=\zeta$. This contradiction finishes our proof. 


\subsection{Proof of Theorem $\mathbf{1 . 6}$}

We first explain our method of describing $n$-gons in $S_{\theta}^{1}$. Let $f: \boldsymbol{Z} \rightarrow \boldsymbol{R}$ be an increasing map such that $f(i+n)=2 \pi \theta+f(i)$ for all $i$. Let $\widetilde{\exp }: \boldsymbol{R} \rightarrow \widetilde{S}^{1}$ be the lift of the exponential map to the universal cover. Let $T_{\theta}$ be the translation of $\widetilde{S}^{1}$ by $2 \pi \theta$, as in $\S 1$. Then $\widetilde{\exp } \circ f: \boldsymbol{Z} \rightarrow \widetilde{S}^{1}$ defines a map which has the following two properties:

- The points $\widetilde{\exp } \circ f(1), \widetilde{\exp } \circ f(2), \widetilde{\exp } \circ f(3)$ etc. occur in order in $\widetilde{S}^{1}$.

- $\widetilde{\exp } \circ f(i+n)=T_{\theta}(\widetilde{\exp } \circ f(i))$ for all $i$.

In other words, $\widetilde{\exp } \circ f(\boldsymbol{Z})$ is naturally the lift of an $n$-gon $W_{f}$ on $S_{\theta}^{1}$.

If $f(i+4)-f(i)<2 \pi$ for all $i$ then $W_{f}$ is tame and $W_{f} \in \mathcal{W}\left(S_{\theta}^{1}, n\right)$. The regular polygon corresponds to the map $\rho$, with the action $\rho(k)=2 \pi \theta k / n$ for all $k$. If $n>4 \theta$ then $\rho(i+4)-\rho(i)<2 \pi$ and $W_{\rho}$ is tame. In this case, small perturbations of $W_{\rho}$ are also tame.

The function $f$ above is determined by $f(1), \ldots, f(n)$. Thus $\mathcal{W}\left(S_{\theta}^{1}, n\right)$ can be identified with an open subset $U \subset \boldsymbol{R}^{n}$. When $n>4 \theta$, this set contains the point $\rho$ corresponding to the regular $n$-gon. We think of $\phi$ as a map from $U$ to $\boldsymbol{R}$.

Since we are only considering tame $n$-gons, we can compute $\phi$ using the ordinary exponential function rather than its lift, $\widetilde{\exp }$. We have

$$
\phi(f)=\prod_{i=1}^{n} \chi(\exp (f(i), f(i+1), f(i+2), f(i+3))) .
$$

As usual with products, it is easier to work with $\log \phi$. It follows from symmetry, or else from an explicit computation, that the gradient $\nabla \phi$ vanishes at $\rho$. Thus, the behavior of $\phi$ in a neighborhood of $\rho$ is determined by the Hessian $H(\rho)$. We can finish the proof of Theorem 1.6 by showing that $H(\rho)$ has a positive eigenvalue.

By symmetry $H(\rho)$ is a circulent matrix. Thus, to compute $H(\rho)$ we need only compute, for instance, the entries $H_{j 3}=\left.\partial_{x_{j}} \partial_{x_{3}} \phi\right|_{\rho}$ for $j=1, \ldots, n$. We do this computation symbolically in the file excess. It turns out that the derivative $\partial_{x_{3}} \phi$ only depends on $x_{1}, x_{2}, x_{3}, x_{4}, x_{5}$. Thus $H_{j 3}=0$ unless $j=1,2,3,4,5$. Also, we have $H_{13}=H_{53}$ and $H_{23}=H_{43}$. Setting $s=2 \pi \theta / n$ we compute

$$
H_{13}=\frac{2 \exp (2 i s)}{(1-\exp (2 i s))^{2}} ; \quad H_{23}=\frac{-2 \exp (i s)}{(1-\exp (2 i s))^{2}}
$$




$$
H_{33}=\frac{4 \exp (i s)(1+\exp (i s)+\exp (2 i s))}{(1-\exp (2 i s))^{2}} .
$$

Though it doesn't look like it, these quantities are all real, and $H_{13}<0$. Setting $A_{s}=-H_{13}>0$ and simplifying we have

$$
A_{s}^{-1} H_{13}=-1 ; \quad A_{s}^{-1} H_{23}=2+2 \cos (s) ; \quad A_{s}^{-1} H_{33}=-2-4 \cos (s) .
$$

Since $s \in(2 \pi / n, \pi / 2)$ there is a positive constant $B_{s}$ such that

$$
\cos (s)=\cos (2 \pi / n)-B_{s} .
$$

Combining Equations 60 and 61 and using the symmetry above we have

$$
\left[\begin{array}{l}
H_{13} \\
H_{23} \\
H_{33} \\
H_{43} \\
H_{53}
\end{array}\right]=A_{s}\left[\begin{array}{c}
-1 \\
2+2 \cos (2 \pi / n) \\
-2-4 \cos (2 \pi / n) \\
2+2 \cos (2 \pi / n) \\
-1
\end{array}\right]+B_{s}\left[\begin{array}{c}
0 \\
-1 \\
2 \\
-1 \\
0
\end{array}\right]
$$

Let $\omega=\exp (2 \pi / n)$. Like all circulent matrices, $H(\rho)$ has eigenvectors

$$
E_{j}=\left(1, \omega^{j}, \omega^{2 j}, \ldots, \omega^{(n-1) j}\right) ; \quad j=1, \ldots, n .
$$

with corresponding eigenvalues

$$
\lambda_{j}=\sum_{i=1}^{n} \omega^{i j} H_{1 i}=\omega^{-2 j} H_{13}+\omega^{-j} H_{23}+H_{33}+\omega^{j} H_{43}+\omega^{2 j} H_{53} .
$$

The second equality uses the circulent property of the matrix.

By symmetry $\lambda_{j}$ is real. Thus, taking $j=1$, we have

$$
\lambda_{1}=A_{s}\left[\begin{array}{c}
-1 \\
2+2 \cos (2 \pi / n) \\
-2-4 \cos (2 \pi / n) \\
2+2 \cos (2 \pi / n) \\
-1
\end{array}\right] \cdot\left[\begin{array}{c}
\cos (4 \pi / n) \\
\cos (2 \pi / n) \\
1 \\
\cos (2 \pi / n) \\
\cos (4 \pi / n)
\end{array}\right]+B_{s}\left[\begin{array}{c}
-1 \\
2 \\
-1
\end{array}\right] \cdot\left[\begin{array}{c}
\cos (2 \pi / n) \\
1 \\
\cos (2 \pi / n)
\end{array}\right]
$$

The first summand in Equation 65 is 0, by basic trigonometry! Therefore

$$
\lambda_{1}=B_{s}(2-2 \cos (2 \pi / n))>0 .
$$

We have found a positive eigenvalue, and our proof is done. 


\section{$7 \quad$ A Modified Process}

The conformal averaging process is natural in the sense that it commutes with all the conformal automorphisms of $S^{1}$, whether they preserve or reverse the orientation of $S^{1}$. We can give up some of this symmetry and ask about processes which commute with orientation preserving conformal symmetries of $S^{1}$. Here we will describe such a process.

Let $W=\left\{w_{1}, \ldots, w_{n}\right\}$ be a polygon in $S^{1}$, as in $\S 1$. For each $j$ there is a unique point $w_{j}^{*}$ such that the geodesic joining $w_{j-1}$ to $w_{j}^{*}$ is perpendicular to the geodesic joinint $w_{j}$ to $w_{j+1}$. Algebraically, $w_{j}^{*}$ is determined by

$$
\chi\left(w_{j-1}, w_{j}, w_{j}^{*}, w_{j+1}\right)=\frac{1}{2} .
$$

We define $W^{*}=\left\{w_{1}^{*}, \ldots, w_{n}^{*}\right\}$. Inductively we define $W^{[k+1]}=\left(W^{[k]}\right)^{*}$. The process $\left\{W^{[k]}\right\}$ is simpler than the process $\left\{W^{(k)}\right\}$ in the sense that each point of $W^{[k+1]}$ is defined in terms of 3 consecutive points of $W^{[k]}$ whereas each point of $W^{(k+1)}$ is defined in terms of 4 consecutive points of $W^{(k)}$. On the other hand, the process $\left\{W^{[k]}\right\}$ is less symmetric than the process $\left\{W^{(k)}\right\}$, as we mentioned above.

After some fooling around we get

$$
w_{j}^{*}=\frac{S\left(\ldots, 0,0,0,0, z_{2}, \ldots, z_{j-1}, \frac{1}{2} z_{j}, 0,0,0 \ldots\right)}{S\left(\ldots, 0,0,0, z_{1}, z_{2}, \ldots, z_{j-1} \frac{1}{2} z_{j}, 0,0,0 \ldots\right)} ; \quad j=1,2,3 \ldots
$$

(When $j=1,2$ this equation must be interpreted as in Equation 69.) Here are the first few terms.

$$
\begin{gathered}
w_{1}^{*}=\frac{1}{1-\frac{1}{2} z_{1}} ; \\
w_{2}^{*}=\frac{1-\frac{1}{2} z_{2}}{1-z_{1}-\frac{1}{2} z_{2}} ; \\
w_{3}^{*}=\frac{1-z_{2}-\frac{1}{3} z_{3}}{1-z_{1}-z_{2}-\frac{1}{2} z_{2}-\frac{1}{2} z_{1} z_{3}} ; \\
w_{4}^{*}=\frac{1-z_{2}-z_{3}-\frac{1}{2} z_{4}+\frac{1}{2} z_{2} z_{4}}{1-z_{1}-z_{2}-z_{3}-\frac{1}{2} z_{4}+z_{1} z_{3}+\frac{1}{2} z_{1} z_{4}+\frac{1}{2} z_{2} z_{4}}
\end{gathered}
$$

Equation 68 has a proof which is quite similar to the proof of Equation 17 given in $\S 2.3$. We omit this proof. 
In the file mapping we compute

$$
\chi\left(w_{1}^{*}, w_{2}^{*}, w_{3}^{*}, w_{4}^{*}\right)=\frac{\left(2+z_{2}\right)\left(2+z_{4}\right) z_{3}}{\left(2+2 z_{2}-z_{2}\right)\left(2+2 z_{3}-z_{4}\right)}
$$

From this formula we see that

$$
\frac{\phi\left(W^{*}\right)}{\phi(W)}=\left[\frac{\prod_{j=1}^{n}\left(2+z_{j}\right)}{\prod_{j=1}^{n}\left(2+2 z_{j}-z_{j+1}\right)}\right]^{2}
$$

As usual, indices are taken $\bmod n$.

We now derive the inequality

$$
\prod_{j=1}^{n}\left(2+z_{j}\right) \geq \prod_{j=1}^{n}\left(2+2 z_{j}-z_{j+1}\right) ; \quad \forall\left(z_{1}, \ldots, z_{n}\right) \in[0,1]^{n},
$$

with equality iff $z_{1}=\ldots=z_{n}$. The proof is almost exactly like the proof for Equation 8 given in $\S 3$. We define

$$
G\left(z_{1}, \ldots, z_{n}\right)=\log \prod_{j=1}^{n}\left(2+z_{j}\right)-\log \prod_{j=1}^{n}\left(2+2 z_{j}-z_{j+1}\right)
$$

Given $Z=(a, b, c, \ldots)$ we compute

$$
G_{b}=\frac{\left(8 b+2 b^{2}\right)-(4 a+4 c+2 a c)}{(2+2 a-b)(2+b)(2+2 b-c)} .
$$

If $\max (a, c) \leq b$ and $\min (a, c)<b$ then $G_{b}>0$. This shows that $G$ attains its global minimum-namely 0 -precisely along the diagonal. This proves Equation 73. At this point, all the arguments in $\S 4.1$ and $\S 4.2$ go through. In particular, we have an alternate proof of Corollary 1.2.

To complete the proof of Theorem 1.1 for the modified process we just need to establish Lemma 3.1 for $G$. We compute that

$$
G_{a b}=\frac{-1}{(2+a)^{2}} ; \quad G_{b b}=\frac{2}{(2+a)^{2}} ; \quad G_{c b}=\frac{-1}{(2+a)^{2}}
$$

Here we have set $(a, b, c)=(a, a, a)$. This computation works for every coordinate, and so $H_{G}$ has the same form as in Equation 31, except with $(2+a)^{-2}$ replacing $(1+2 a)^{-2}$. Thus, Lemma 3.1 remains true.

Now that we have assembled all the ingredients, the proofs of Theorems $1.1,1.3,1.4$, and 1.5 go through essentially word for word. 


\section{References}

[B] A. F. Beardon, The Geometry of Discrete Groups (1983) Springer

[K] N.H. Kuiper, Locally Projective Spaces of Dimension One, Mich. Math. Journal 2 (1953-54) 95-97

[S] R. Schwartz A Projectively Natural Flow for Circle Diffeomorphisms, Inventiones Mathematicae 110 (1992) pp. 627-647

[W] S. Wolfram, Mathematica: A System for Doing Mathematics by Computer, Wolfram Press (2000) 